\title{
Anti-HSP47 siRNA lipid nanoparticle ND-L02-s0201 reverses interstitial pulmonary fibrosis in preclinical rat models
}

\author{
Yun Liu ${ }^{1,3}$, Jian Liu ${ }^{1,3}$, Alistair Quimbo ${ }^{1}$, Fengcheng Xia ${ }^{1}$, Jiping Yao ${ }^{1}$, \\ Jean-Pierre Clamme ${ }^{1}$, Sonya Zabludoff ${ }^{1}$, Jun Zhang ${ }^{2}$ and Wenbin Ying ${ }^{1}$
}

Affiliations: ${ }^{1}$ Nitto Biopharma Inc., San Diego, CA, USA. ${ }^{2}$ Cellagen Technology, San Diego, CA, USA. ${ }^{3}$ These authors contributed equally.

Correspondence: Wenbin Ying, Nitto Biopharma Inc., 10618 Science Center Drive, San Diego, CA 92121, USA E-mail: wenbin.yinglanitto.com

ABSTRACT ND-L02-s0201 is a lipid nanoparticle encapsulating an siRNA which inhibits expression of heat shock protein 47 (HSP47), a collagen-specific chaperone. Accumulated evidence demonstrates a close association between increased level of HSP47 and excessive accumulation of collagen in fibrotic diseases. Our objective was to test ND-L02-s0201 efficacy in preclinical lung fibrosis models and characterise the downstream histological and functional consequences of inhibiting the expression of HSP47.

Comprehensive optimisation and characterisation of bleomycin (BLM) and silica-induced rat lung fibrosis models were conducted, which ensured progressive pathological changes were sustained throughout the study during evaluation of the anti-fibrotic potential of ND-L02-s0201.

In the BLM model, we demonstrated dose-dependent and statistically significant reduction in the relative lung weight, collagen deposition and histology, and fibrosis scores following ND-L02-s0201 treatment. Lung tissue mRNA profiling demonstrated that 11 out of 84 fibrosis-relevant genes were upregulated following BLM induction and were downregulated by approximately 4.5-fold following NDL02-s0201 treatment. Epithelial-mesenchymal transition was characterised in the BLM model following ND-L02-s0201 treatment. Cell enrichment demonstrated that myofibroblasts contained the highest HSP47 mRNA expression. BLM led to more than a five-fold increase in myofibroblasts and ND-L02-s0201 treatment reduced the myofibroblasts to sham levels. Statistically significant improvement in lung function was noted in the BLM model which was determined by running endurance capacity using a 7-minute treadmill test. Comparable anti-fibrotic efficacy was also observed in the silica model.

Results from two robust chronic rodent models of pulmonary fibrosis demonstrated significant antifibrotic effects and improved lung function which support the evaluation of ND-L02-s0201 in subjects with idiopathic pulmonary fibrosis.

@ERSpublications

By targeting HSP47 and facilitating normalisation of EMT, siRNA lipid nanoparticle ND-L02s0201 reverses interstitial pulmonary fibrosis, restores structural integrity of the lung, and improves pulmonary function in preclinical rat models. https://bit.ly/3a2lo1B

Cite this article as: Liu Y, Liu J, Quimbo A, et al. Anti-HSP47 siRNA lipid nanoparticle ND-L02s0201 reverses interstitial pulmonary fibrosis in preclinical rat models. ERJ Open Res 2021; 7: 00733 2020 [https://doi.org/10.1183/23120541.00733-2020].

This article has supplementary material available from openres.ersjournals.com.

Received: 22 Oct 2020 | Accepted: 19 Jan 2021

Copyright $\odot$ The authors 2021. This version is distributed under the terms of the Creative Commons Attribution NonCommercial Licence 4.0. For commercial reproduction rights and permissions contact permissions@ersnet.org 


\section{Introduction}

Idiopathic pulmonary fibrosis (IPF) is a specific form of chronic, progressive fibrotic interstitial pneumonia of unknown cause. The prevalence increases with age and the pathological changes are limited to the lungs. IPF is characterised by progressive worsening of dyspnoea and lung function and is associated with a poor prognosis [1]. The median survival of idiopathic pulmonary fibrosis patients is generally 2-3 years after diagnosis. Respiratory failure resulting from disease progression is the most frequent cause of death [2].

Advances have been made in the past decades in understanding the pathogenesis of IPF. Initially IPF was considered as the result of chronic inflammation, but current evidence suggests IPF may be caused by an abnormal wound-healing process. Repeated alveolar epithelial cell injury or apoptosis, with abnormal re-epithelialisation, continuous fibroblast to myofibroblast transition, and progressive extracellular matrix (ECM) accumulation, result in lung tissue destruction and fibrosis, eventually leading to respiratory compromise and failure [3,4]. Myofibroblasts as a key cell type, are involved in fibrosis progression in response to signals of tissue damage, tissue remodelling, and wound repair through the regulated deposition and maintenance of the ECM components [5]. In addition to fibroblasts [6, 7], other cell types including bone marrow-derived circulating pluripotent mesenchymal progenitor (fibrocyte) [8] and endothelial [9] have been demonstrated to play important roles in myofibroblast recruitment and enrichment through the epithelial-to-mesenchymal transition (EMT) or endothelial-to-mesenchymal transition (EndMT) mechanisms. The existence of multiple-sources of myofibroblast recruitment and sustained myofibrobalst activation, proliferation, and survival results ultimately in impaired tissue repair due to excessive ECM synthesis and deposition.

Heat shock protein 47 (HSP47) is a collagen-specific molecular chaperone residing in the endoplasmic reticulum, which is essential for the correct folding of procollagen in the endoplasmic reticulum. The expression of HSP47 under normal conditions correlates with collagens in various cell types and tissues and also helps to maintain haemostasis and the integrity of tissue structure. Additionally, HSP47 has been reported to play a pivotal role in pathological conditions such as fibrotic diseases, which include excessive collagen accumulation and deposition within interstitial space. Elevation of HSP47 expression has been observed in multiple forms of fibrosis [10-12]. Preclinical studies demonstrate that downregulation of HSP47 through RNA interference (RNAi) resulted in decrease in collagen deposition, amelioration of fibrosis, and restoration of organ function in different disease models, such as liver fibrosis [13], pulmonary fibrosis [14], skin fibrosis [15], and dry eye syndrome in chronic graft versus host disease (GVHD) [16].

Immunohistochemistry demonstrated the co-expression of HSP47 and procollagen (type I) in the pneumocytes (type II) and myofibroblasts in patient samples with usual interstitial pneumonia. Evidence has revealed the increased expression of HSP47 in fibrotic lesions of IPF and other pulmonary disorders with diffuse alveolar damage [17]. HSP47 was detected in the serum of patients with acutely exacerbated IPF [18]. Myofibroblasts, through the production of HSP47-associated regulation of type I procollagen, play an important role in the progression of pulmonary fibrosis [19]. However, direct evidence supporting the involvement of HSP47 in the EMT process and its role in the development and progression of lung fibrosis is not available. The present study evaluates whether HSP47 regulates the EMT process during formation of fibrotic tissue and disease progression in rat lung fibrosis models.

ND-L02-s0201 is a lipid nanoparticle (LNP) formulation that incorporates six key lipid components, to encapsulate the active pharmaceutical ingredient (API), NDT-05-0038, which is a nuclease resistant, synthetic, double stranded small interfering ribonucleic acid (siRNA) designed to reversibily inhibit the expression of HSP47 by targeting the homologous sequence across human, rat, and mouse. The LNP includes a retinoid-conjugated targeting agent (di-retinamide-PEG-di-retinamide), which promotes uptake by target cells (hepatic stellate cells for liver fibrosis or lung myofibroblasts for IPF). Multiple clinical trials have evaluated the safety, tolerability, and pharmacokinetics of ND-L02-s0201 in healthy volunteers and in subjects with liver fibrosis. In general, ND-L02-s0201 was well tolerated in the five completed clinical studies (three in healthy subjects $[20,21]$ and two in subjects with liver fibrosis [22]. Biological activities were observed with improvements in efficacy studies in progressive hepatic fibrosis (METAVIR F3-4) noted in METAVIR, Ishak and Knodell fibrosis scores, as well as decreased stiffness by FibroScan at week 5 [22]. In 2018, JUNIPER: a phase II study to evaluate the safety, biological activity, and PK of ND-L02-s0201 in subjects with IPF was initiated due to the encouraging early signs of efficacy in advanced liver fibrosis, preclinical efficacy in IPF models, and significant unmet medical need in IPF patients [23].

We describe here the preclinical efficacy of ND-L02-s0201 in lung fibrosis resolution in several animal models with demonstrated progressive fibrosis over a range of study durations. These studies evaluated the effect of knockdown of the target gene, HSP47, on lung fibrosis resolution through biochemical and histological examinations as well as functional recovery through treadmill assessments. 


\section{Materials and methods}

\section{ND-L02-s0201}

ND-L02-s0201 was manufactured to contain six key lipids in the composition, including the cationic, helper, and targeting lipids which encapsulates the API siRNA (NDT-05-0038). Particles were prepared using an ethanol injection process by combining an ethanolic solution of the lipids with an aqueous solution of siRNA. The final buffer composition of ND-L02-s0201 consisted of NDT-05-0038/lipid nanoparticles in $20 \mathrm{mM}$ HEPES with a $9 \%$ sucrose buffer. Lipid composition and their respective molar ratios, siRNA:lipid ratio were developed to optimise drug loading capacity, potency, and product safety. The effect of each process parameter on key drug product quality attributes such as particle size, stability, and nucleic acid encapsulation, as well as in vitro and in vivo efficacy was determined. All the dosing concentrations in the studies described for ND-L02-s0201 refer to the concentration of NDT-05-0038.

\section{Animals}

Sprague-Dawley rats (250-280 g) were purchased from Charles River Laboratories. The animals were housed in standard caging systems under an alternating 12 -h light/dark cycle, temperature at $18-26^{\circ} \mathrm{C}$ and humidity at $30-70 \%$. Animals were provided certified standard fresh rodent chow and tap water ad libitum. All animal involving procedures were approved by the Institutional Animal Care and Use Committees (IACUC) at Explora Biolabs (San Diego, CA, USA).

\section{Rat model of bleomycin-induced lung fibrosis}

The animal body weight was 300-350 g at the initial BLM treatment. BLM (APP Pharmaceuticals, LLC. Schaumberg, IL, USA) was diluted in $0.9 \%$ saline for oropharyngeal aspiration at a final concentration of $6 \mathrm{mg} \cdot \mathrm{mL}^{-1}$ or $2 \mathrm{mg} \cdot \mathrm{mL}^{-1}$ for $3 \mathrm{mg} \cdot \mathrm{kg}^{-1}$ or $1 \mathrm{mg} \cdot \mathrm{kg}^{-1}$ per application. BLM induction was initiated at $3 \mathrm{mg} \cdot \mathrm{kg}^{-1}$ on day 0 , followed by $1 \mathrm{mg} \cdot \mathrm{kg}^{-1}$ on days $1-6$ for the first week. Subsequent BLM boost was conducted at $3 \mathrm{mg} \cdot \mathrm{kg}^{-1}$ once on day 14 for the 5-week model or five times on days 14, 28, 42, 56 and 70 for the 10-week model [23].

\section{Rat model of silica-induced lung fibrosis}

Min-U-Sil5 silica powder (a gift from US Silica Corporation, Frederick, MD, USA) was suspended in $0.9 \%$ saline for oropharyngeal aspiration at the final concentration of $83.3 \mathrm{mg} \cdot \mathrm{mL}^{-1}$. Silica was administrated at $25 \mathrm{mg}$ per $\mathrm{g}$ of lung [25] on days $0,1,2,14,28$, and 42 . Silica induction was conducted using same procedure described above for BLM induction. Silica suspension was mixed thoroughly before each administration.

\section{Collagen content}

Quantification of lung collagen was conducted following a modified procedure [26]. A slice of lung tissue from right interior lobe (100-150 mg of wet weight) was used in the hydroxyproline assay. Briefly, the lung slices were hydrolysed in $6 \mathrm{~N}$ hydrochloride $(\mathrm{HCl})$ at $110^{\circ} \mathrm{C}$ overnight followed by incubation with 4-dimethylamino-benzaldehyde for 25 minutes at $65^{\circ} \mathrm{C}$, and absorbance of oxidised hydroxyproline was determined at $558 \mathrm{~nm}$. The amount of collagen was calculated based on standard curve generated in parallel and expressed in micrograms per lung based on the total wet lung weight.

\section{Primary cell isolation/enrichment and characterisation}

Isolation of subsets of different cell types was conducted following the procedure with certain modifications $[27,28]$. Briefly, alveolar macrophages were collected by repeated intratracheal lavage with $5 \mathrm{mM}$ EDTA in D-PBS following cardiac perfusion. Isolation/enrichment of subpopulations of endothelial, epithelial or myofibroblast were achieved through gradient centrifugation following enzymatic digestion. Characterisation of each cell populations was conducted through quantitative polymerase chain reaction (Q-PCR) with gene-specific probes, EPCAM (Rn01473202_m1) for epithelial, ICAM2 (Rn01461346_m1) for endothelial, ACTA2 (Rn01759928_g1) for myofibroblast and PTPRC (CD45) (Rn00709901_m1) for macrophage (Thermo-Fisher, Waltham, MA, USA).

A subset of animals from each group (six from sham, five from BLM and six from BLM+ND-L02-s0201) were used for correlation assessment between fibrotic severity and enrichment of different cell populations. Left lungs were collected for histopathology evaluation and right lungs for cell fractionation.

\section{Real-time PCR}

Quantification of HSP47 through real-time polymerase chain reaction (RT-PCR) was conducted following the instruction provided by the manufacturer. Total ribonucleic acid (RNA) was extracted from a section of right caudal lobe of lung tissue or from isolated cell fractions described above using RNeasy columns (Qiagen, Germantown, MD, USA) and adjusted to $100 \mathrm{ng} \cdot \mu \mathrm{L}^{-1}$. Real-time PCR was performed on the ViiA7 system in triplicate using the TaqMan Gene Expression Master Mix (Applied Biosystems, Foster 
City, CA, USA). The relative gene expression was presented as fold-change of each gene of interest (GOI) compared with the sham group (designated as 1) for the following GOIs: ACTA2 (Rn01759928_g1), EPCAM (Rn01473202_m1), ICAM2 (Rn01461346_m1), and PTPRC (CD45) (Rn00709901_m1), Serpinh1 (HSP47, Rn0567777_m1), MRPL19 (Rn01425270_m1).

\section{Rat fibrosis $R T^{2}$ profiler PCR array}

Satellite animals from each group were used for gene expression analysis of genes involved in fibrosis initiation and development. Six rats were selected from the BLM-treated alone group (fibrotic score 3-4, trichrome staining) and six rats from the BLM+ND-L02-s0201 group (fibrotic score 1), and four rats from the sham group as control. A panel of 84 key fibrosis related genes were assessed with rat Fibrosis $\mathrm{RT}^{2}$ Profiler PCR Arrays Kit (Qiagen, Germantown, MD, USA). Briefly, the first strand cDNA was synthesised with $100 \mathrm{ng}$ total RNA in a $20 \mu \mathrm{L}$ reaction volume with $\mathrm{RT}^{2}$ First Strand Kit (Qiagen, Germantown, MD, USA) and followed by amplification with $\mathrm{RT}^{2}$ SYBR Green Q-PCR Master Mix (Qiagen, Germantown, MD, USA) in a 384-well plate format. Raw data was exported and analysed using the integrated web-based automated software for $\mathrm{RT}^{2}$ profiler PCR Array data analysis which was available through Qiagen (www. qiagen.com/us/shop/genes-and-pathways/data-analysis-center-overview-page).

\section{Histopathology}

Left lung tissues were fixed with phosphate-buffered saline (PBS) neutralised 10\% paraformaldehyde, sectioned $(4-6 \mu \mathrm{m})$ and processed with haematoxylin and eosin $(\mathrm{H} \& \mathrm{E})$ or Masson's trichrome stain for histopathology assessment at Wax-it Histology Services, Inc. (Vancouver, BC, Canada). The slides were evaluated by a US Board-certified pathologist at Nova Pathology (Vancouver) in a semi-quantitative manner, including key parameters of atelectasis, bronchiectasis, alveolar/interstitial oedema, alveolar emphysema, pulmonary fibrosis, alveolar haemorrhage, hyperplasia of type II alveolar epithelial cells, epithelial hypertrophy/hyperplasia of distal airways, alveolar macrophage infiltration and interstitial/airway inflammation, with severity score based on the following system: minimal $=1$, mild $=2$, moderate $=3$ and marked $=4$.

\section{Epithelial-mesenchymal transition study}

Single cell suspensions from naïve Sprague-Dawley rat (100-200 g) lung tissue were produced through enzymatic digestion and gradient centrifugation following the procedure with certain modifications [27, 28]. The resulting cell pellets were panned on rat immunoglobulin $\mathrm{G}$ pre-coated petri dish at $37^{\circ} \mathrm{C}$ for 30 minutes. Unattached cells were collected,rinsed and seeded on collagen pre-coated $10 \mathrm{~cm}$ dishes in the medium of Dulbecco's modified Eagle medium-containing $10 \%$ fetal bovine serum, at $1 \times 10^{6} \mathrm{~mL}^{-1}$. Following the initial 48 hours, cells were fractionated into $75 \mathrm{~mm}^{2}$ flasks at $2 \times 10^{6} /$ flask. BLM induction was conducted 4 -h following seeding on day 0 at $2 \mathrm{mg} \cdot \mathrm{mL}^{-1}$. After $24 \mathrm{~h}$ the cell cultures were switched to freshly prepared medium supplemented with $50 \mathrm{nM}$ ND-L02-s0201 and cultured for another 2 hours. Afterwards the cell cultures were switched back to the fresh medium containing $0.2 \mathrm{mg} \cdot \mathrm{mL}^{-1} \mathrm{BLM}$ for additional designated period. Characterisation of cell population following the treatments was conducted on days 0,3 , 4, 5 and 7 through fluorescence-activated cell sorting (FACS) (Accuri C6, BD Biosciences) using immunostaining with anti $\alpha$-smooth muscle actin (SMA)-fluorescein isothiocyanate (Abcam, Cambridge, UK) and anti E-cadherin-Alexa 647 (BD Biosciences, San Jose, CA, USA).

\section{Treadmill training and testing}

Treadmill training and testing were conducted on the treadmill device (Cat\# LE8710RTS, Panlab/Harvard Apparatus, Holliston, MA, USA). Animal qualification for the treadmill test was the completion of running distance $>20 \mathrm{~m}$ for the last minute at the belt speed at $60 \mathrm{~cm} \cdot \mathrm{s}^{-1}$ during a 7 -min training session. A total of 30 rats selected from training session were randomised into three groups, ten rats were assigned to the sham group, and the other 20 rats were assigned for BLM induction. More than $80 \%$ pre-trained sham group animals were able to complete the total 7-min running duration at $60 \mathrm{~cm} \cdot \mathrm{s}^{-1}$ with no signs of exhaustion. SEDACOM software (Panlab/Harvard Apparatus, Holliston, MA, USA) was used to visualise and export the data of the running capacity for further analysis.

\section{Data analysis}

Statistical analysis was performed for assays which contained three or more groups $(n \geqslant 3)$, using one-way ANOVA followed by Dunn's multiple comparisons post hoc test to determine if there were statistically significant differences observed between the mean or median values between groups. Unless otherwise specified, multigroup analysis evaluated treatment groups as compared to vehicle groups. For assays in which two groups were compared, non-paired t-test was used for statistical analysis. For all analyses, a $\mathrm{p}$-value $<0.05$ was considered statistically significant $\left({ }^{*}: \mathrm{p}<0.05 ;{ }^{* *}: \mathrm{p}<0.01 ;{ }^{* *}: \mathrm{p}<0.001\right.$; and $\left.{ }^{* * *}: \mathrm{p}<0.0001\right)$. 


\section{Results}

ND-L02-s0201 treatment resulted in resolution of lung fibrosis in optimised BLM model in rats The aim of this study was to evaluate the therapeutic effects of ND-L02-s0201 in an optimised BLM-induced rat model. BLM induction followed the protocol of EGGER et al. [29] with certain modifications to avoid significant spontaneous recovery and to ensure the persistence of formation of fibrotic tissue throughout the study. Validation of the BLM model was conducted through assessments of relative lung weight, whole hydroxyproline content, and histopathological analysis (supplementary figure 1). In the BLM model shown in supplementary figure 1A, compared with the sham group (open bar), repeated oropharyngeal aspiration of BLM in the vehicle group resulted in severe lung damage reflected by increased relative lung weight (supplementary figure 1B), elevated collagen (hydroxyproline content) deposition in lung tissue (supplementary figure 1C), and substantial increase in histopathological scores (supplementary figure 1D). Excessive collagen deposition was further confirmed through trichrome staining (supplementary figure 1E) at the end of the study (day 49), 5 weeks following the final dose of BLM on day 14

BLM-treated animals received ND-L02-s0201 treatment intravenously at an initial dose of $2 \mathrm{mg} \cdot \mathrm{kg}^{-1}$ on Day 18 and followed by subsequent weekly doses at $2,1.5,1$, or $0.5 \mathrm{mg} \cdot \mathrm{kg}^{-1}$. ND-L02-s0201 treatment resulted in statistically significant fibrosis resolution in a dose-dependent manner, including restoration of relative lung weight, reduction of hydroxyproline content and histological and trichrome scores (figure 1b-e). Single initial dose of $2 \mathrm{mg} \cdot \mathrm{kg}^{-1}$ followed by $1.5 \mathrm{mg} \cdot \mathrm{kg}^{-1}$, weekly for 4 weeks led to statistically significant lung fibrosis resolution.

Gene array analysis demonstrated up regulation of a set of genes involved in collagen synthesis/deposition and fibrosis formation following BLM induction. ND-L02-s0201, at an initial dose of $2 \mathrm{mg} \cdot \mathrm{kg}^{-1}$ followed by $1.5 \mathrm{mg} \cdot \mathrm{kg}^{-1}$, weekly for 4 weeks, led to the reversal of the increase in the expression of these genes in addition to the siRNA target gene HSP47 (figure $1 \mathrm{~g}$ and $\mathrm{h}$ ). Q-PCR results for the comparison of the full panel of 84 genes in the array are shown in supplementary table 1.

\section{Cellular update of ND-L02-s0201}

Characterisation of cellular update was conducted with fluorescence-labelled ND-L02-s0201 in BLM rat model. Colocalisation assessment demonstrates that formulation promotes the uptake into myofibroblasts as well as additional cell types like alveolar type- 2 cells which can transform into myofibroblasts (supplementary figure 2).

\section{Downregulation of $\alpha$-SMA and HSP47 co-expression following ND-L02-s0201 treatment in lung tissue from BLM model}

To evaluate the effect of HSP47 downregulation on $\alpha$-SMA, image quantification analyses of the anti-HSP47 or $\alpha$-SMA immunohistochemistry staining from the sequential sections of lung tissue from the study presented in figure 1 was conducted. As shown in supplementary figure 8, BLM induction in the vehicle group caused substantial elevation in both HSP47 and $\alpha$-SMA expression and ND-L02-s0201 treatment led the reductions in both HSP47 and $\alpha$-SMA (supplementary figure 8A and B). Representative images are presented in supplementary figure 8. Image alignment of anti-HSP47 and $\alpha$-SMA staining from the sequential sections demonstrates clear co-localisation between them. Furthermore, there was a good correlation between the two staining $\left(\mathrm{R}^{2}=0.6162, \mathrm{p}<0.0001\right)$ (supplementary figure $8 \mathrm{C}$ ).

\section{Reversal of BLM-induced accumulation of myofibroblasts and accelerated EMT through downregulation of HSP47}

Ex vivo evaluations were conducted to further understand the roles of different cell types during fibrotic formation and progression using primary cells from BLM treated rat lung tissue. Four different cell types (i.e. myofibroblast, endothelial, epithelial, and macrophage) were enriched and characterised through specific gene marker analysis (supplementary figure 3). Q-PCR data demonstrated myofibroblasts showed the highest level of HSP47 expression among the four cell types. BLM induction did not result in the elevation of HSP47 mRNA levels in any cell types (figure 2a). Instead, increases in cell number were observed following BLM induction, more prominently in myofibroblasts (more than five-fold) and less significantly among other cell types (supplementary figure 4). Correlation analysis demonstrated a statistically significant relationship between myofibroblast cell numbers and lung weight with $\mathrm{R}^{2}=0.556$ (supplementary figure 6). ND-L02-s0201 treatment resulted in substantial reduction of myofibroblast counts towards the level of the sham group (figure $2 \mathrm{~b}$ ). The myofibroblast cell counts also correlated to the tissue fibrosis scores through correlation statistical analysis $\left(R^{2}=0.688, n=6\right)$ (figure 2c). Dose-dependent downregulation of HSP47 in the primary myofibroblasts was observed following ND-L02-s0201 treatment with an estimated half-maximal effective concentration of $13 \mathrm{nM}$ (figure 2d). 


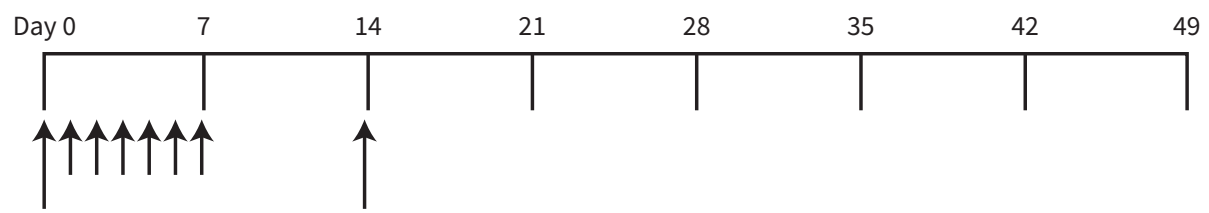

$3 \mathrm{mg} \cdot \mathrm{kg}^{-1}+1 \mathrm{mg} \cdot \mathrm{kg}^{-1} \times 6+$
$3 \mathrm{mg} \cdot \mathrm{kg}^{-1}$ on day $14(\mathrm{OP})$
$\Delta$

b)

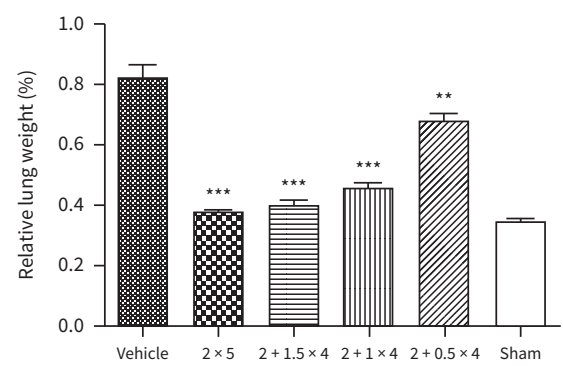

e)

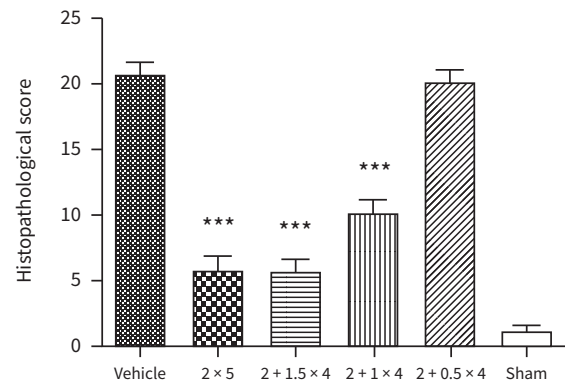

g)

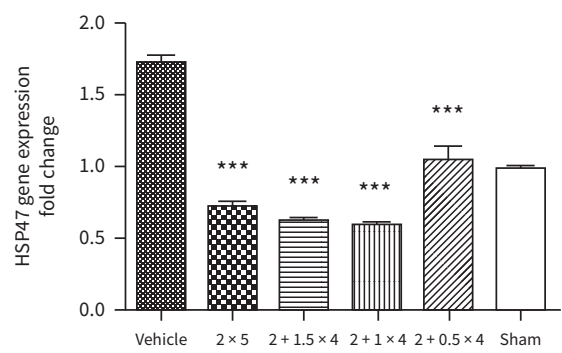

f)

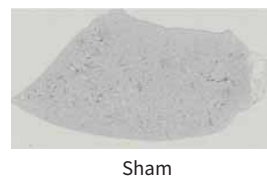

h) c)

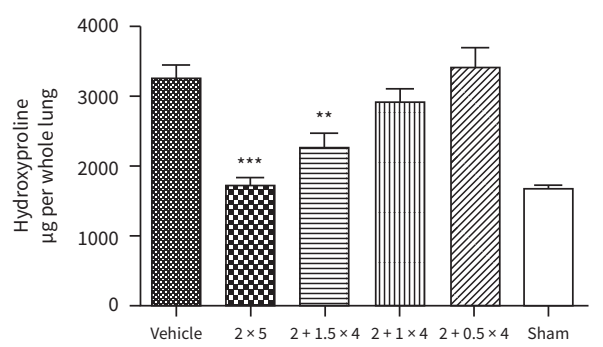

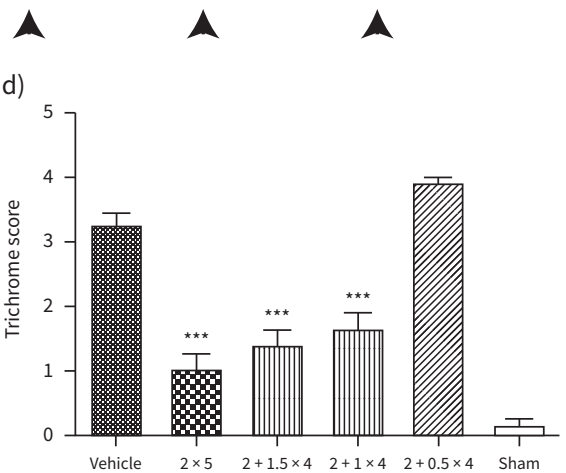
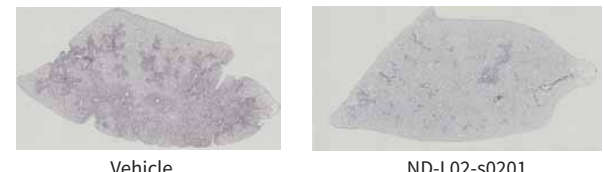

ND-L02-50201

$2 \mathrm{mg} \cdot \mathrm{kg}^{-1}$ per week, $\mathrm{x} 5$

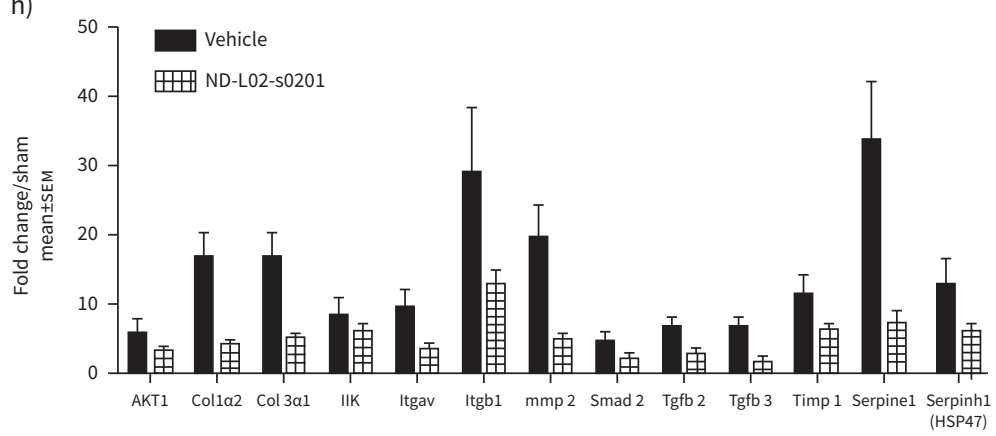

FIGURE 1 In vivo efficacy of ND-L02-s0201 in bleomycin (BLM)-induced chronic lung fibrosis model in rats. a) Model illustration. Male Sprague-Dawley rats were treated with BLM eight times at $3 \mathrm{mg} \cdot \mathrm{kg}^{-1}$ (Day 0,14$)$ and $1 \mathrm{mg} \cdot \mathrm{kg}^{-1}$ (Day 1 to Day 6) (0.5 mL $\cdot \mathrm{kg}^{-1}$ ) to induce chronic lung damage. ND-L02-s0201 was dosed i.v. with initial dose of $2 \mathrm{mg} \cdot \mathrm{kg}^{-1}$ on Day 18 and weekly follow-up doses at $0.5,1,1.5,2 \mathrm{mg} \cdot \mathrm{kg}^{-1}$ for another 4 weeks. Two control groups were included for comparisons: 1) animals not treated with BLM or siRNA formulation (Sham, $\mathrm{n}=8$ ); 2) animals induced with BLM but not treated with siRNA formulation (Vehicle, n=12). b) Relative lung weight. Three days after last dose of siRNA treatment, lungs were harvested and weighed during necropsy and relative lung weights were calculated based on the body weight of each animal on necropsy day. c) Lung hydroxyproline content. A section of the right lung lobe from each animal was collected and stored at $-80^{\circ} \mathrm{C}$ until tissue lysis for hydroxyproline quantification. $d$ and e) Histopathology evaluation. The left lung was perfused and inflated and fixed for tissue section and haematoxylin and eosin $(H \& E)$ and trichrome staining. Histological findings were assigned a semiquantitative severity score based on the following system: minimal=1, mild=2, moderate=3 and marked=4, either independent score of trichrome stain (d) or sum of a panel of parameters of H\&E stain (e) (refer to Materials and Methods, Histopathology section for details). f) Representative images (trichrome stain) following ND-L02-s0201 treatment (2 mg. $\mathrm{kg}^{-1} \cdot$ week $^{-1}, 4$ weeks) in BLM rat lung. g) Downregulation of target gene HSP47 following ND-L02-s0201 treatment. h) Representative Q-PCR results from rat Fibrosis RT2 Profiler PCR Arrays (Qiagen) consisting of a panel of 84 key genes involved in fibrosis. $2 \times 5: 2 \mathrm{mg} \cdot \mathrm{kg}^{-1}$ for 5 weeks; $2+1.5 \times 4: 2 \mathrm{mg} \cdot \mathrm{kg}^{-1}$ initial then $1.5 \mathrm{mg} \cdot \mathrm{kg}^{-1}$ for 4 weeks; $2+1 \times 4: 2 \mathrm{mg} \cdot \mathrm{kg}^{-1}$ initial then $1 \mathrm{mg} \cdot \mathrm{kg}^{-1}$ for 4 weeks; $2+0.5 \times 4: 2 \mathrm{mg} \cdot \mathrm{kg}^{-1}$ initial then $0.5 \mathrm{mg} \cdot \mathrm{kg}^{-1}$ for 4 weeks. ${ }^{* *}: \mathrm{p}<0.01 ;{ }^{* * *}: \mathrm{p}<0.001$.

Influence of HSP47 on EMT was further evaluated in primary epithelial cells from lung tissue. As shown in figure 2e, epithelial cells displayed a natural course of EMT in culture by Day 3-7 with reduced E-cad positive cells (solid line, filled circle) and increased $\alpha$-SMA positive cells (dotted line, open circle). EMT 
a)

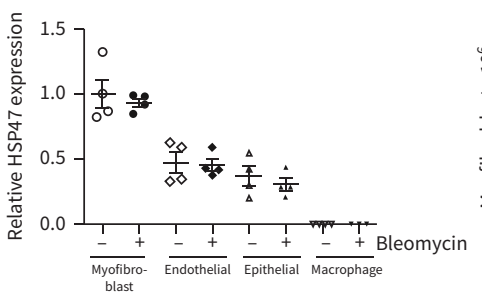

e)

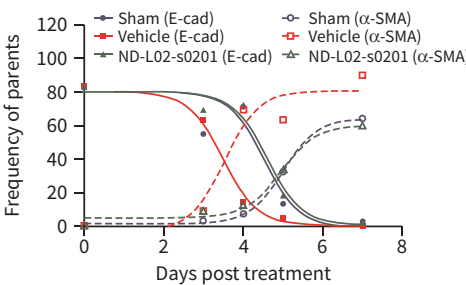

b)

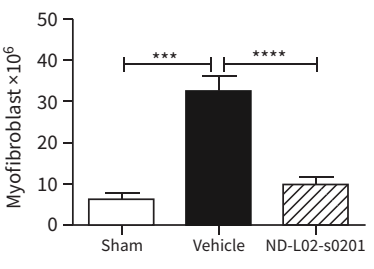

f)

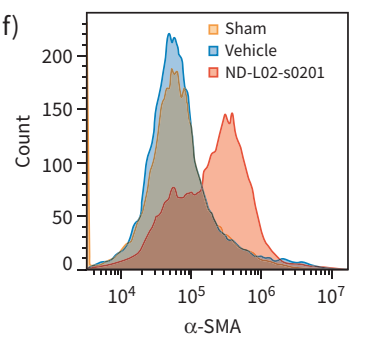

c)
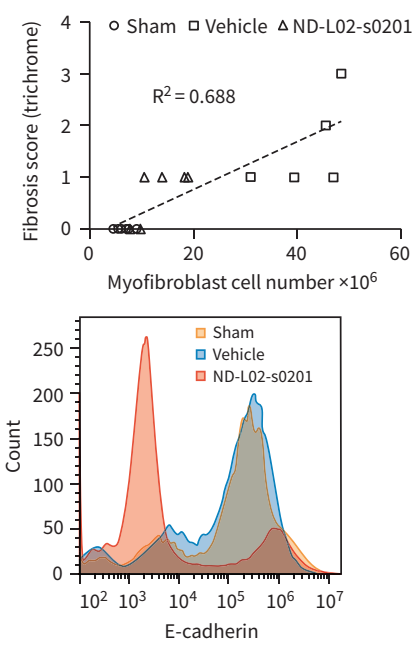

d)

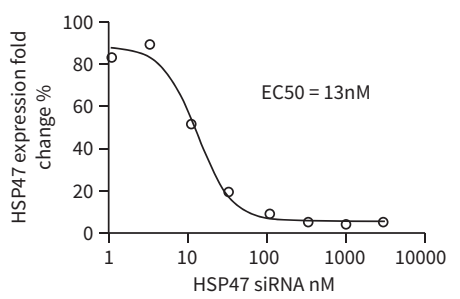

FIGURE 2 Epithelial-to-mesenchymal transition (EMT) ex vivo studies following bleomycin (BLM) induction and ND-L02-s0201 treatment. a) Q-PCR quantification of HSP47 from four cell types isolated from rat lungs with (+) and without $(-)$ BLM induction as illustrated in figure 1a. b) Total cell counts for myofibroblast cells isolated from rat lungs following respective treatment in BLM-induced IPF model. c) Correlation analysis between the fibrosis scores of trichrome stain (left lungs) and the myofibroblast cell counts (right lungs) of the same animals following different treatment ( $\mathrm{n}=6$ ): Sham, BLM+Vehicle, and BLM+ND-L02-s0201. BLM induction and ND-L02-s0201 treatment were illustrated in figure 1a. d) Dose-dependent HSP47 gene knock down for primary rat myofibroblast cells isolated from BLM-treated animals following $24 \mathrm{~h}$ culture post a 30 min transfection incubation with ND-L02-s0201 (1 nM-3 $\mu$ M). e) Primary lung epithelial cells were exposed to BLM and subjected to ND-L02-s0201 treatment as well. Double staining of $\alpha$-smooth muscle actin (SMA) and E-cadherin (E-Cad) positive cells were analysed through fluorescence-activated cell sorting (FACS) on Day $0,3,4,5$, and 7 post BLM initiation. Frequencies of parent cells were plotted for E-cad positive (filled symbols) and $\alpha$-SMA positive cells (open symbols). f) FACS histograms analysis on day 4 for cells analysed in (e).

process was accelerated following BLM induction evidenced by left shifting of both E-cad and $\alpha$-SMA curves. This acceleration was reversed following $24 \mathrm{~h}$ treatment with $50 \mathrm{nM}$ ND-L02-s0201 (figure 2f). Complete FACS analysis of all cell samples on Days 0, 3, 4, 5, 6, and 7 following BLM and ND-L02-s0201 treatment are shown in supplementary figure 5.

\section{ND-L02-s0201 treatment in chronic rodent models of IPF demonstrates statistically significant improvement in fibrosis}

Therapeutic effects of ND-L02-s0201 in a clinically preferred once every two weeks dosing frequency were investigated in comparison with weekly dosing regimen in both chronic BLM model and the silica-induced fibrotic model.

In the chronic bleomycin model, additional four BLM inductions were conducted on days 28, 42, 56 and 70. Anti-fibrotic effects of ND-L02-s0201 were assessed in an extended duration of 12 weeks with the regimen either weekly (ten total doses) or once every two weeks (five total doses) (figure 3a). Compared with the sham group, the lung fibrotic changes in the vehicle group were maintained through the extended period (hydroxyproline content and histopathology parameters). As presented in figure $3 \mathrm{~b}-\mathrm{d}$, a trend towards dose-dependent decrease in hydroxyproline content and histopathology improvement were observed following ND-L02-s0201 treatment in comparison to the vehicle group. Similar therapeutic potency was achieved at once every two weeks dosing regimen in comparison to weekly dosing regimen when an approximate total drug was given (figure $3 \mathrm{e}-\mathrm{g}$ ). There were no statistical differences in total hydroxyproline content and trichrome scores between groups, once every two weeks, $3 \mathrm{mg} \cdot \mathrm{kg}^{-1} \times 5$ weeks compared with weekly, $2 \mathrm{mg} \cdot \mathrm{kg}^{-1} \times 10$ weeks or weekly, $3 \mathrm{mg} \cdot \mathrm{kg}^{-1} \times 10$ weeks $(\mathrm{p}>0.05$, t-test, two-tailed).

Silicosis, a common form of occupational lung disease, is caused by inhalation of crystalline silica dust and leads to inflammation, fibrosis, and nodular legions in the lung. As shown in figure 4, a silica-induced lung fibrotic model was developed in rats with repeated OP aspiration of silica suspension of $25 \mathrm{mg}$ per $\mathrm{g}$ of lung. Following silica administration, animals in Vehicle Group showed progressively enlarged lungs in a time-course dependent manner from day 21-101 as well as alterations in other relevant fibrotic parameters (figure $4 \mathrm{~b}-\mathrm{e}$ ).

Anti-fibrotic effects of ND-L02-s0201 in the silica model was evaluated at $2 \mathrm{mg} \cdot \mathrm{kg}^{-1}$, weekly with different treatment durations (figure $4 \mathrm{f}-\mathrm{i}$ ). Even though the numerical reduction of hydroxyproline level did not reach 


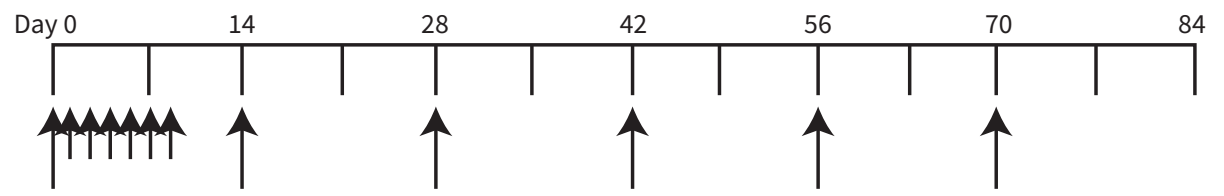

Bleomycin

ND-L02-s0201 regimen \#1

ND-L02-s0201 regimen \#2
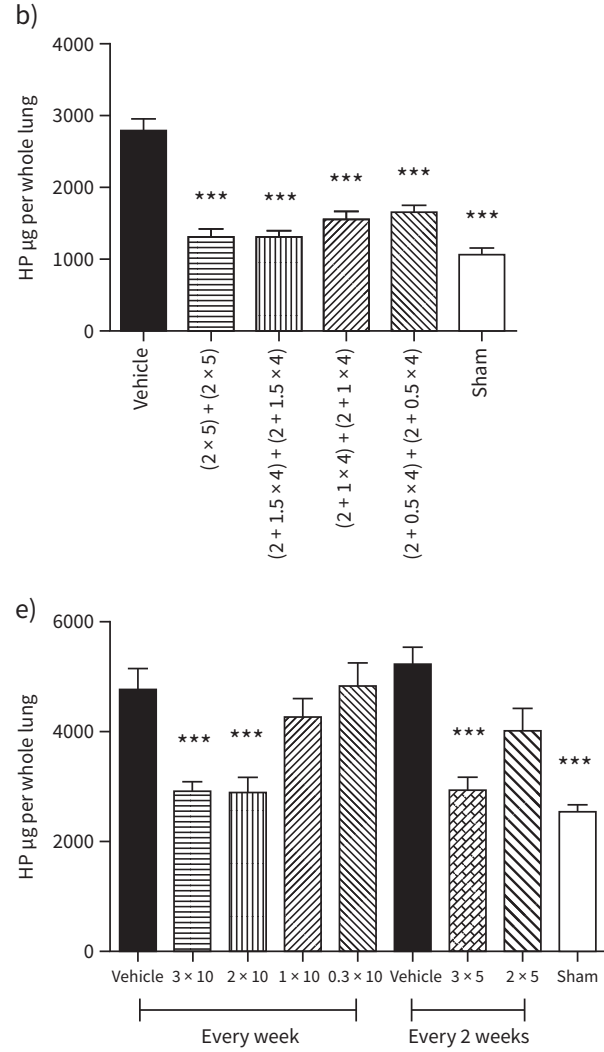

c)

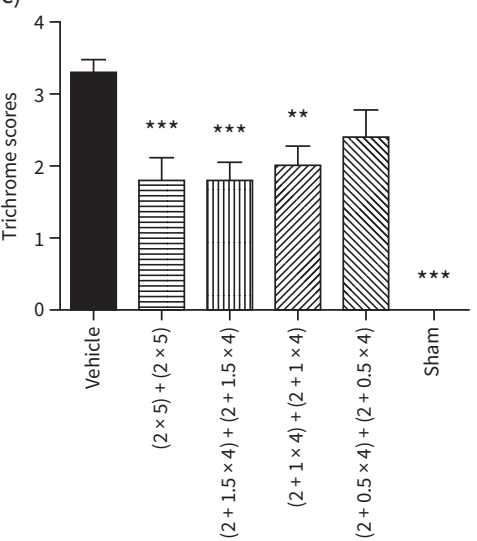

f)

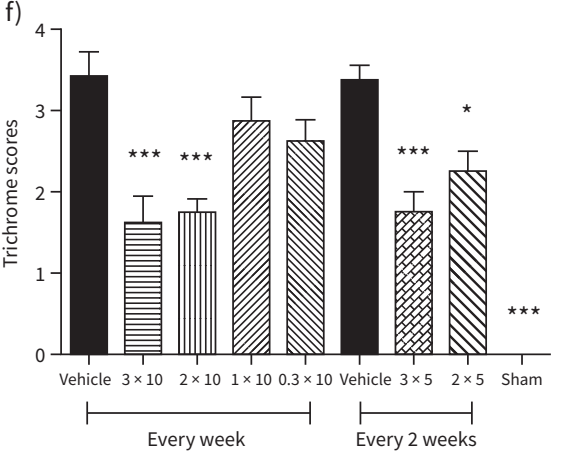

d)

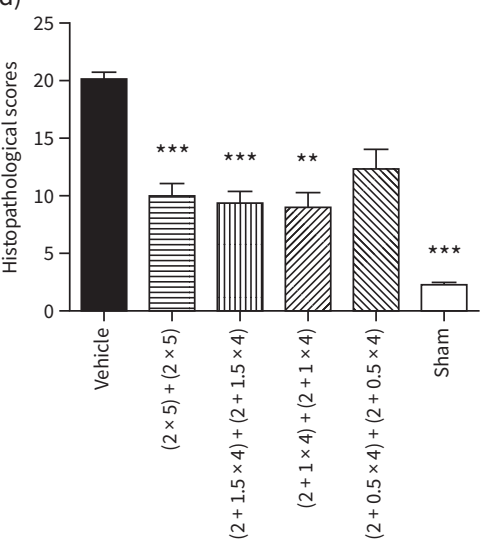

g)

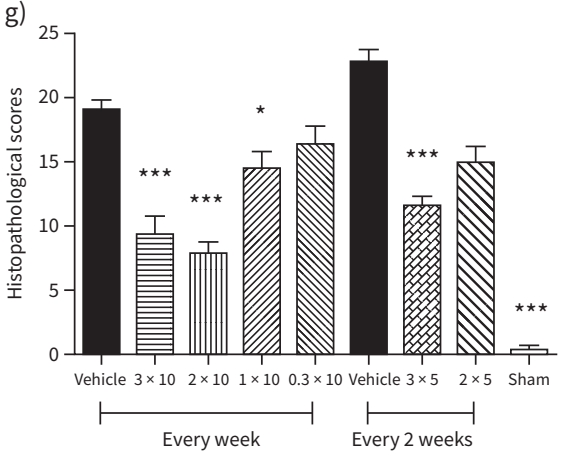

FIGURE 3 Modified extended bleomycin (BLM) model with revised dosing schedule. a) Model illustration. Male Sprague-Dawley rats were treated with BLM 12 times at $3 \mathrm{mg} \cdot \mathrm{kg}^{-1}$ (Day $0,14,28,42,56,70$ ) and $1 \mathrm{mg} \cdot \mathrm{kg}^{-1}$ (Day 1 to day 6) $\left(0.5 \mathrm{~mL} \cdot \mathrm{kg}^{-1}\right.$ ) to induce chronic lung damage. ND-L02-s0201 was dosed i.v. weekly or once every two weeks for 10 weeks starting from day 18 . b-d) Animals were subjected to weekly ND-L02-s0201 treatment, two cycles of 5 -week treatments: one initial dose of $2 \mathrm{mg} \cdot \mathrm{kg}^{-1}$ plus four follow up doses of $0.5-2 \mathrm{mg} \cdot \mathrm{kg}^{-1}$. $2 \times 5$ : $2 \mathrm{mg} \cdot \mathrm{kg}^{-1}$ for 5 weeks; $2+1.5 \times 4: 2 \mathrm{mg} \cdot \mathrm{kg}^{-1}$ initial then $1.5 \mathrm{mg} \cdot \mathrm{kg}^{-1}$ for 4 weeks; $2+1 \times 4: 2 \mathrm{mg} \cdot \mathrm{kg}^{-1}$ initial then $1 \mathrm{mg} \cdot \mathrm{kg}^{-1}$ for 4 weeks; $2+0.5 \times$ 4: $2 \mathrm{mg} \cdot \mathrm{kg}^{-1}$ initial then $0.5 \mathrm{mg} \cdot \mathrm{kg}^{-1}$ for 4 weeks. e-g) Animals were subjected to ND-L02-s0201 treatments, either weekly at doses of 0.3-3 mg.kg $\mathrm{kg}^{-1}$ for 10 doses or once every two weeks at doses of 2 and $3 \mathrm{mg} \cdot \mathrm{kg}^{-1}$ for five doses. Lung hydroxyproline (HP) content (b and e), trichrome score ( $\mathrm{c}$ and $\mathrm{f}$ ), and histology score ( $\mathrm{d}$ and $\mathrm{g}$ ) were performed as described in figure 1 following necropsy on Day 84 . Statistically significant difference was based on comparing to corresponding vehicle group. ${ }^{*}: p<0.05 ;{ }^{* *}: p<0.01 ;{ }^{* * *}: p<0.001$.

statistical significance, all treatment groups showed remarkable decreases in histopathological and trichrome scores. Comparison between once every two weeks and weekly dosing regimens demonstrated a similar anti-fibrotic potency at equivalent total dosage of $20 \mathrm{mg} \cdot \mathrm{kg}^{-1}$ over a 10 -week duration $\left(4 \mathrm{mg} \cdot \mathrm{kg}^{-1} \times 5\right.$ weeks or $2 \mathrm{mg} \cdot \mathrm{kg}^{-1} \times 10$ weeks), reflected by reduction in hydroxyproline level and improvement in histopathology and trichrome scores and the restoration of relative lung weight (figure $4 \mathrm{j}$ and $\mathrm{k}$ ).

\section{ND-L02-s0201 treatment leads to the recovery of pulmonary function in BLM-treated rats}

Impact of ND-L02-s0201 on pulmonary function in the BLM-induced rats was assessed by evaluating the running endurance on a treadmill. Animals were stratified into two groups, sham and BLM, following the training phase targeting equivalent ability to consistently complete the treadmill testing at $60 \mathrm{~cm} \cdot \mathrm{s}^{-1}$ with the final minute of running covering a distance exceeding $20 \mathrm{~m}$ (figure 5a). BLM induction resulted in significant reduction in total running distance starting day 16 as compared to the sham group, demonstrating an impact on lung function. BLM-treated rats were further stratified into two groups 
a)

Silica induction

ND-L02-s0201 regimen = 1

ND-L02-s0201 regimen $=2$

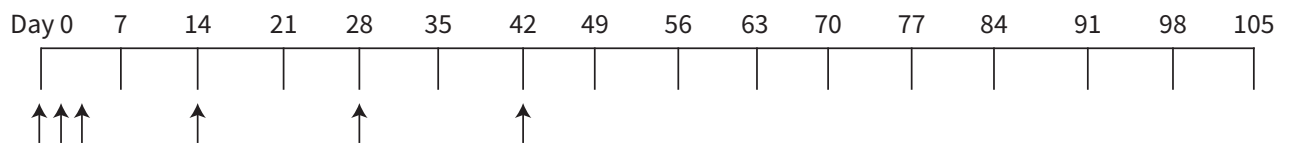

b)

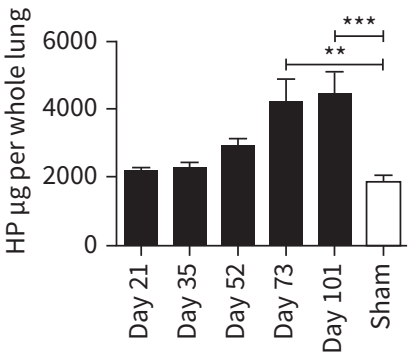

f)
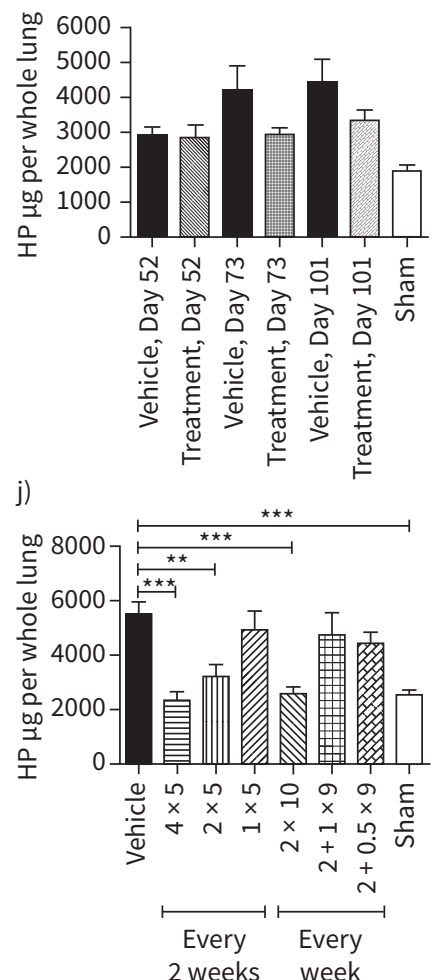

c)

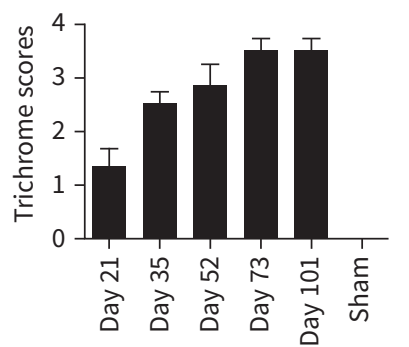

g)

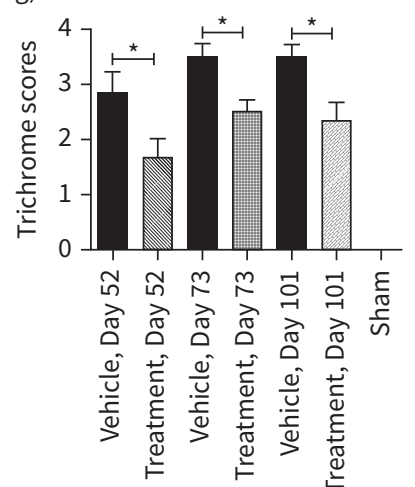

k)

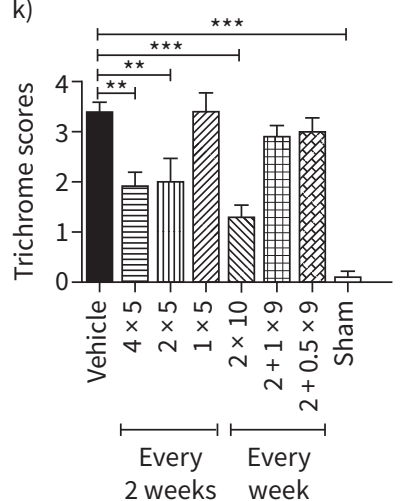

d)

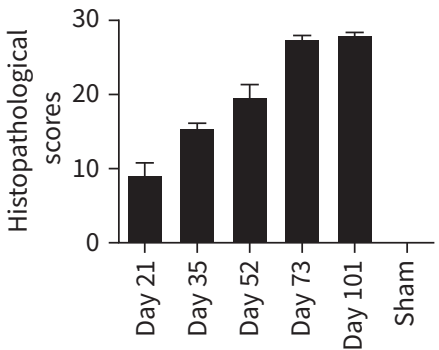

h)
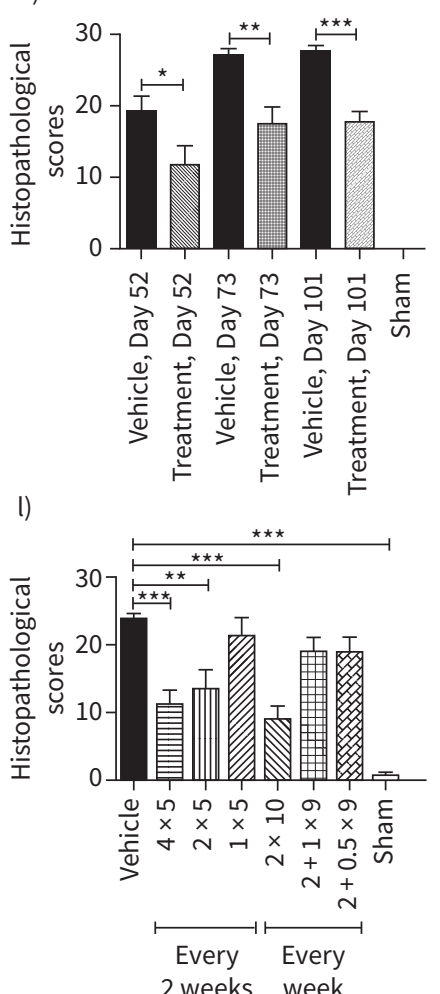
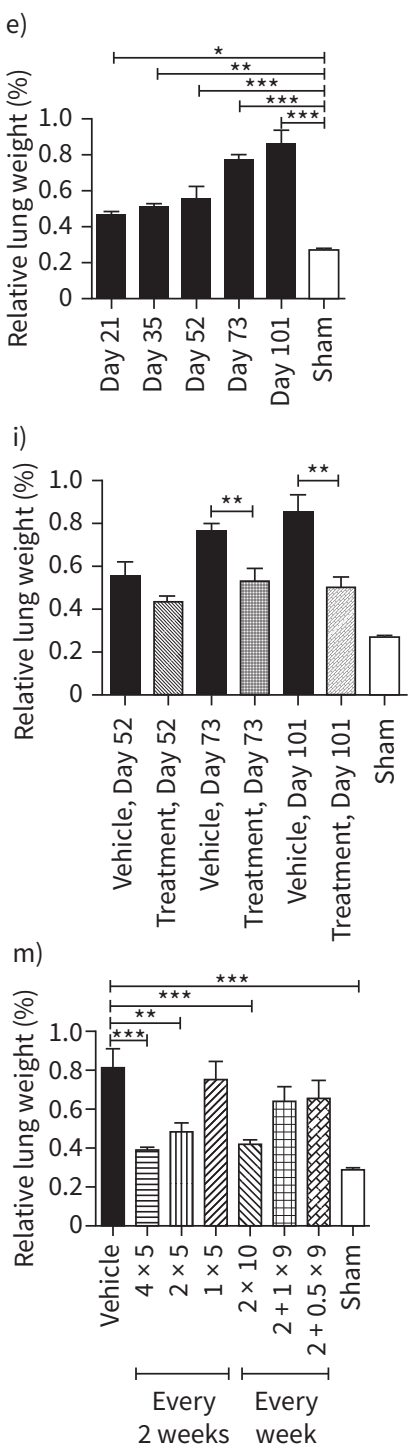

FIGURE 4 In vivo efficacy of ND-L02-s0201 in silica-induced chronic lung fibrosis model in rats. a) Model illustration. Male Sprague-Dawley rats were treated with silica six times at $25 \mathrm{mg}$ per $\mathrm{g}$ of lung (Day $0,1,2,14,28$, and 42) to induce chronic lung damage. ND-L02-s0201 was dosed i.v. weekly or once every two weeks for 10 weeks. Lung hydroxyproline (HP) content (b, f and j), trichrome score (c, g and k), histology score (d, h and l) and relative lung weight ( $\mathrm{e}, \mathrm{i}$ and $\mathrm{m}$ ) were performed as described in figure 1 following necropsy on respective days. b-e) Silica treated animals were monitored on Days 21, 35, 52, 73, and 101 to monitor lung damage progression. $f-i)$ Three study durations were compared with termination on Days 52, 73, and 101 to evaluate ND-L02-s0201 efficacy. j-m) ND-L02-s0201 treatment were completed once every two weeks at 4, 2, and $1 \mathrm{mg} \cdot \mathrm{kg}^{-1}$ for five doses or with an initial dose at $2 \mathrm{mg} \cdot \mathrm{kg}^{-1}$ starting on Day 35 plus nine weekly follow up doses at 2,1, and $0.5 \mathrm{mg} \cdot \mathrm{kg}^{-1}$. $4 \times 5$ : $4 \mathrm{mg} \cdot \mathrm{kg}^{-1}$ for 5 doses; $2 \times 5: 2 \mathrm{mg} \cdot \mathrm{kg}^{-1}$ for 5 doses; $1 \times 5: 1 \mathrm{mg} \cdot \mathrm{kg}^{-1}$ for 5 doses; $2 \times 10: 2 \mathrm{mg} \cdot \mathrm{kg}^{-1}$ for 10 weeks; $2+1 \times 9: 2 \mathrm{mg} \cdot \mathrm{kg}^{-1}$ initial then $1 \mathrm{mg} \cdot \mathrm{kg}^{-1}$ for 9 weeks; $2+0.5 \times 9: 2 \mathrm{mg} \cdot \mathrm{kg}^{-1}$ initial then $0.5 \mathrm{mg} \cdot \mathrm{kg}^{-1}$ for 9 weeks. Animal lungs were examined on Day 101 follow standard procedures described above. ${ }^{*}: p<0.05 ;{ }^{* *}: p<0.01 ;{ }^{* * *}: p<0.001$.

(vehicle and ND-L02-s0201 treatment) based on day 16 data (figure 5b). ND-L02-s0201 treatment was initiated on day 18 at $2 \mathrm{mg} \cdot \mathrm{kg}^{-1}$, weekly for 4 weeks. As shown in figure $5 \mathrm{~d}$, the vehicle group animals showed further decline in running capacity on days 18-23 and sustained poor running endurance 
a) Naïve rats $(n=40)$
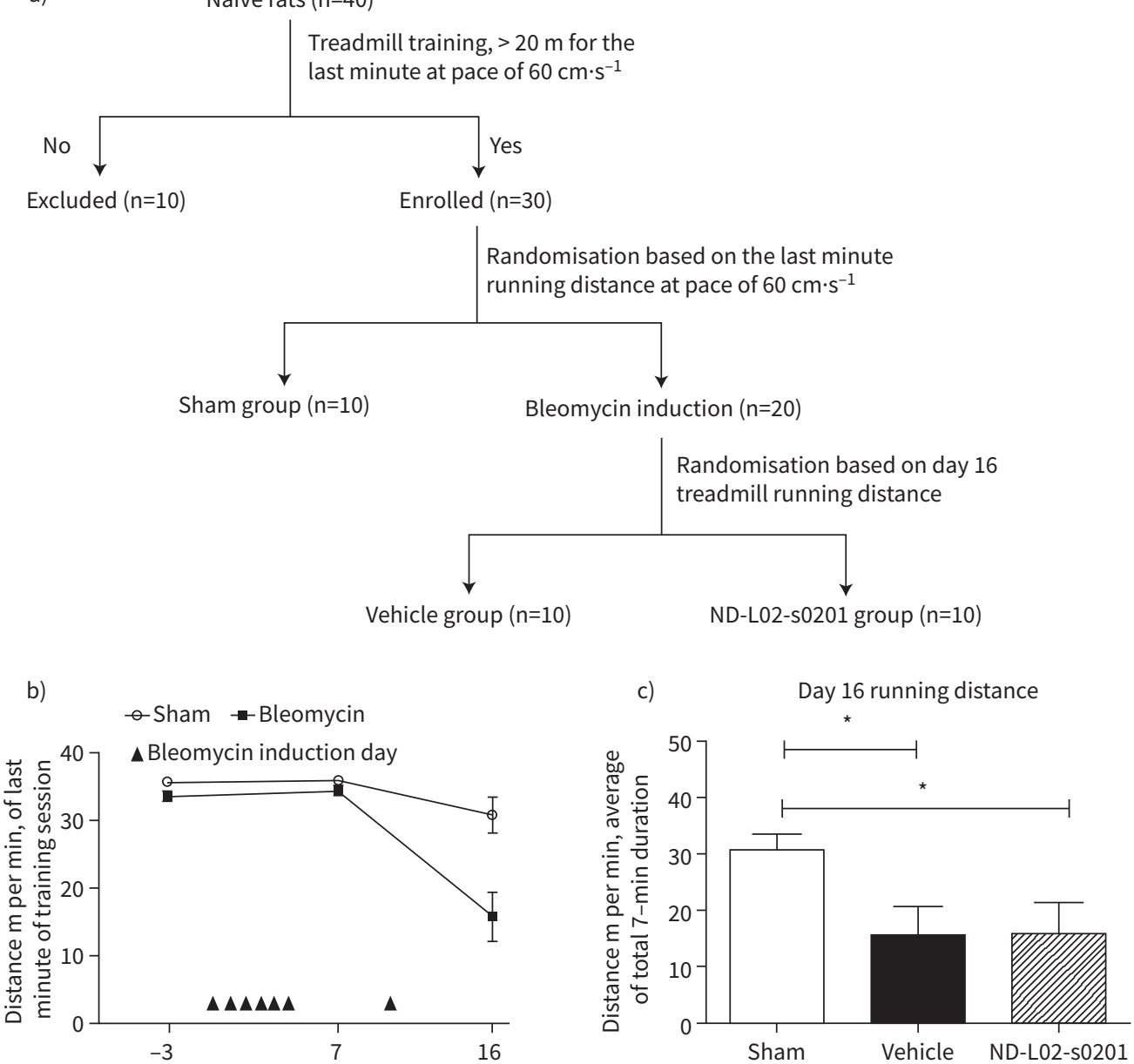

Day post initial bleomycin induction

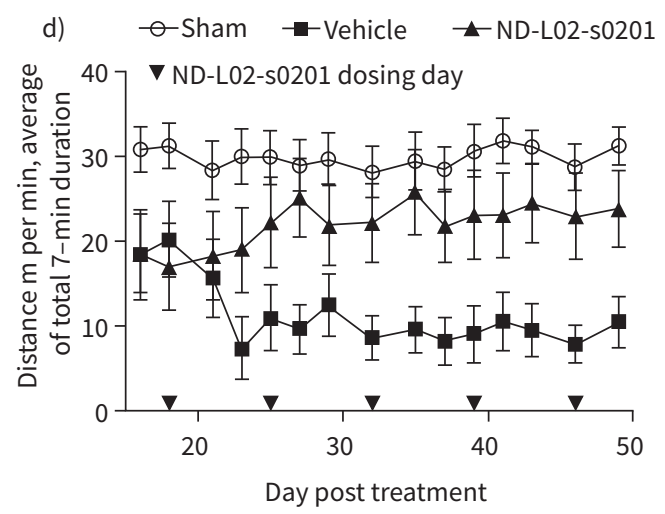

e)

Day 49 running distance

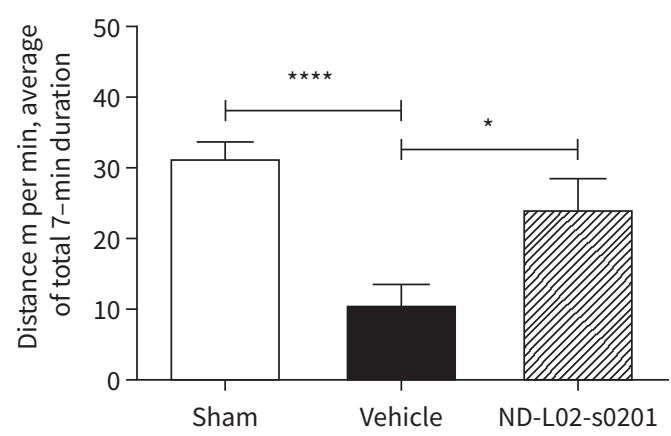

FIGURE 5 Running endurance evaluation following bleomycin (BLM) induction in rat chronic model of lung fibrosis. a) Animal stratification chart following treadmill training and BLM induction. b) Running distance before and after BLM induction, prior to ND-L02-s0201 treatment initiation. Last minute running distance recorded on Days 3, 7, and 16 for animals with or without BLM inductions las indicated by triangles on Days 0-6 and Day 14, same regimen as figure 1a). c) Randomisation of BLM-treated animals into Vehicle and ND-L02-s0201 treatment group ( $\mathrm{n}=10$ each), based on Day 16 treadmill running distance. d) Average running distance per minute of total 7-min test duration at a speed of $60 \mathrm{~cm} \cdot \mathrm{s}^{-1}$ during siRNA treatment period (Days 18-49). ND-L02-s0201 $2 \mathrm{mg} \cdot \mathrm{kg}^{-1}$ treatment was delivered weekly starting on Day 18 and continued for 4 weeks. Treadmill tests were conducted three times per week for animals not treated with BLM (Sham), treated with BLM (Vehicle), and treated with BLM and ND-L02-s0201 (ND-L02-s0201). e) Final treadmill running test result on Day 49. *: $p<0.05 ;{ }^{* * *}: p<0.0001$. 
throughout the study. Animals receiving ND-L02-s0201 showed improved performance on the treadmill for the remaining testing period. At day 49, the final test showed significant reduction in running capacity of animals in the vehicle group compared with the sham group $(\mathrm{p}<0.0001$, unpaired $\mathrm{t}$-test), confirming the detrimental impact of BLM induction on the lung function. Animals following ND-L02-s0201 treatment demonstrated statistically significant improvement in running capacity compared with the vehicle group $(\mathrm{p}<0.05)$. There was no statistically significant difference between the ND-L02-s0201 treatment and sham groups $(\mathrm{p}>0.05)$ (figure $5 e)$.

The treadmill test has been implemented as a key parameter for cardiopulmonary function assessment to evaluate the degree of impairment in the heart, lung, or skeletal muscle system. In considering the local (oropharyngeal) administration of BLM in this study and the lack of BLM-related cardiac or skeletal muscle dysfunction which were assessed by an independent board-certified pathologist, we believe the improvement in running endurance following the treatment of ND-L02-s0201 was mainly due to the resolution of pulmonary functions rather than improvement in other unlikely toxicities caused by BLM treatment. There was no substantial body weight loss among the groups towards the end course of the treadmill tests (supplementary figure 7). These results provide strong evidence that ND-L02-s0201 treatment can benefit the pulmonary function recovery in the BLM induced lung fibrosis model.

\section{Discussion}

In viewing the limited number of therapeutic options that can slow disease progression and the progressive course of the illness, there exists a tremendous unmet medical need for highly effective and well-tolerated agents that halt, or ideally reverse, the fibrosis in IPF patients. Progress in the understanding of the pathophysiology has led to the development of novel experimental therapeutics. For example, inhibition of aberrant fibroblasti proliferation and accumulation of extracellular matrix proteins, such as collagen, have become the focus of current therapeutic studies for IPF [30]. HSP47 is a key molecular chaperone that facilitates collagen triple helix formation and is critical for collagen formation. Studies utilising pulmonary fibrosis clinical samples which demonstratedan increase in HSP47 expression in lung tissue and plasma $[17-19,31]$ as well as colocalisation of HSP47 and type I procollagen in type II pneumocytes and myofibroblasts in usual interstitial pneumonia [19], led to great interest in developing novel therapeutics through inhibition of HSP47 as an approach to control aberrant production of collagen [32].

ND-L02-s0201 is a drug product designed to downregulate HSP47 specifically and result in the reduction of collagen synthesis and deposition. In the present study, ND-L02-s0201 treatment led to substantial reduction of HSP47,a decrease in hydroxyproline content,improvement of histological parameters, andrestoration of relative organ weight in rat fibrotic models. Unsurprisingly, a series of pro-fibrotic genes were upregulated following BLM induction, which are relevant to collagen synthesis/secretion, and fibrosis development. Following ND-L02-s0201 treatment, the increases in gene expression were reversed, driven by inhibition of HSP47 (named SERPINH1 in the array, figure 1h, supplementary table 1). Further investigations need to be conducted to address the mechanisms of the regulation of pro-fibrotic genes through HSP47 machinery.

Serving as a key source of excess production of collagenous ECM [33], myofibroblasts are fundamental drivers in the development and progression of fibrosis in IPF and therefore are of great interest in both research and development of novel therapeutics for IPF treatment. Multiple sources of myofibroblast recruitment and accumulation have been identified. Additionally, activated myofibroblasts demonstrate augmented proliferation and resistance to programmed cell death [34].

During EMT, the epithelial cells express distinct mesenchymal markers and lose epithelial markers as well as apicobasal polarity and intercellular junctions. Alteration of cell polarity and adhesion results in the disruption of basement membrane and facilitates epithelial cell penetration of the ECM-rich compartment [35]. In the present study, the increase in the myofibroblast population was more prominent compared with all other cell types. This increase was positively correlated to the relative lung weight gain as well as severity of the fibrotic score. In vitro assay with primary rat epithelial cells exposed to BLM showed facilitation of the transition from epithelial to myofibroblast phenotype. Further analysis demonstrated myofibroblasts had the highest HSP47 expression which was not increased further following BLM treatment. The elevated expression of HSP47 in the lung following BLM treatment was mainly attributed to the increase in myofibroblast numbers instead of the expression level in the cells. Downregulation of HSP47 through ND-L02-s0201 treatment successfully prevented the progression of EMT both in vivo and in vitro. To our knowledge, this is the first study that provides direct evidence that inhibition of HSP47 can prevent progression of lung fibrosis through EMT suppression. It has been reported by other laboratories that downregulation of HSP47 resulted in endoplasmic reticulum stress-mediated apoptosis of fibroblasts in cell culture [36, 37], which could also be the mechanism of anti-fibrotic effects of ND-L02-s0201. 
BLM-induced pulmonary fibrosis models have been widely used in preclinical studies of potential therapies development for IPF. Unfortunately, the drug candidates through BLM model assessment were unsuccessful due to inappropriate study settings. From 1980 to 2006, the majority of the studies (211 out of 221) were conducted in a post-exposure preventive regimen, in which the test drugs were given $<7$ days after the last BLM dose [38]. It was also reported that spontaneous resolution of fibrosis occurred following a single BLM treatment and infrequent BLM treatment [38, 39]. To support ND-L02-s0201 pharmacological evaluation, we have developed a robust BLM model with optimised induction regimen through full assessments of tissue collagen deposition and histopathological endpoints. Substantial and persistent changes of lung fibrosis were up to 8 weeks through multiple rounds of BLM administration in the chronic BLM models, which provided a sustained evaluation window for reliable efficacy studies. Dose-dependent anti-fibrotic effects of ND-L02-s0201 in this model were observed with weekly dosing, reflected by restoration of relative lung weight, reduction in collagen content, and amelioration in histopathological scores. In addition to the restoration of the structural integrity of lung tissue, improvement of pulmonary function was also observed following ND-L02-s0201 treatment in the running endurance capacity test.

Anti-fibrotic effects of ND-L02-s0201 were observed in a silica-induced lung fibrosis model in rats. Following silica oropharyngeal induction, the increase in lung hydroxyproline content and histopathological changes sustained over 3 months without significant recovery. Intervention with ND-L02-s0201 resulted in amelioration of histopathological alterations and stopped the worsening of the fibrosis. As in the BLM model, ND-L02-s0201 treatment once every two weeks resulted in similar anti-fibrotic potency as a weekly dosing regimen in silica models. Data from both models, either BLM or silica, provide strong evidence supporting once every two weeks dosing of ND-L02-s0201 in IPF patients.

In conclusion, the results from the current studies provide evidence that downregulation of HSP47 expression, a key protein supporting synthesis and deposition of properly folded collagen during lung tissue remodelling following injury, can inhibit the development of fibrosis, stop progression of disease, and help restore pulmonary function. In addition to the direct role on collagen processing, downregulation of HSP47 results in inhibition of EMT which leads to a reduction in myofibroblasts. Anti-fibrotic effects of ND-L02-s0201 have been demonstrated in both BLM-induced and silica-treated rat models, evidenced by the recovery of structural integrity of lung tissue as well as the improvement of pulmonary functions. Given the significant unmet medical need and the preclinical studies here as well as previous clinical studies in healthy volunteers and subjects with hepatic fibrosis, ND-L02-s0201 is now under evaluation in a Phase II clinical study in patients with IPF [23]. Further investigation can be conducted on ND-L02-s0201 as therapeutic option for pulmonary fibrosis arising from different causes including viral infection such as coronavirus disease 2019 [40].

Conflict of interest: Y. Liu is currently an employee of Nitto BioPharma Inc., a subsidiary division of Nitto Denko Tech Corp. J. Liu is currently an employee of Nitto BioPharma Inc., a subsidiary division of Nitto Denko Tech Corp. A. Quimbo is currently an employee of Nitto BioPharma Inc., a subsidiary division of Nitto Denko Tech Corp. F. Xia is currently an employee of Nitto BioPharma Inc., a subsidiary division of Nitto Denko Tech Corp. J. Yao is currently an employee of Nitto BioPharma Inc., a subsidiary division of Nitto Denko Tech Corp. J-P. Clamme is currently an employee of Nitto BioPharma Inc., a subsidiary division of Nitto Denko Tech Corp. S. Zabludoff is currently an employee of Nitto BioPharma Inc., a subsidiary division of Nitto Denko Tech Corp. J. Zhang used to be an employee of Nitto BioPharma Inc., a subsidiary division of Nitto Denko Tech Corp. W. Ying is currently an employee of Nitto BioPharma Inc., a subsidiary division of Nitto Denko Tech Corp.

\section{References}

1 Raghu G. An official ATS/ERS/JRS/ALAT statement: idiopathic pulmonary fibrosis: evidence-based guidelines for diagnosis and management. Am J Respir Crit Care Med 2011; 183: 788-824.

2 Ley B, Collard H, King TJ. Clinical course and prediction of survival in idiopathic pulmonary fibrosis. Am J Respir Crit Care Med 2011; 183: 431-440.

3 Selman M, King T, Pardo A. Idiopathic pulmonary fibrosis: prevailing and evolving hypotheses about its pathogenesis and implications for therapy. Ann Intern Med 2001; 134: 136-151.

4 Thannickal V, Toews G, White E, et al. Mechanisms of pulmonary fibrosis. Annu Rev Med 2004; 55: 395-417.

$5 \quad$ Klingberg F, Hinz B, White E. The myofibroblast matrix: implications for tissue repair and fibrosis. J Pathol 2013; 229: 298-309.

6 Willis B, Liebler J, Luby-Phelps K, et al. Induction of epithelial-mesenchymal transition in alveolar epithelial cells by transforming growth factor-betal: potential role in idiopathic pulmonary fibrosis. Am J Pathol 2005; 166: $1321-1332$.

7 Willis B, duBois R, Borok Z. Epithelial origin of myofibroblasts during fibrosis in the lung. Proc Am Thorac Soc 2006; 3: 377-382.

8 Reilkoff R, Bucala R, Herzog E. Fibrocytes: emerging effector cells in chronic inflammation. Nat Rev Immunol 2011; 11: 427-435.

9 Hashimoto N, Phan S, Imaizumi K, et al. Endothelial-mesenchymal transition in bleomycin-induced pulmonary fibrosis. Am J Respir Cell Mol Biol 2010; 43: 161-172. 
10 Masuda H, Fukumoto M, Hirayoshi K, et al. Coexpression of the collagen-binding stress protein HSP47 gene and the alpha 1(I) and alpha 1(III) collagen genes in carbon tetrachloride-induced rat liver fibrosis. J Clin Invest 1994; 94: 2481-2488.

11 Razzaque MS, Taguchi T. Collagen-binding heat shock protein (HSP) 47 expression in anti-thymocyte serum (ATS)-induced glomerulonephritis. J Pathol 1997; 183: 24-29.

12 Honzawa $\mathrm{Y}$, Nakase $\mathrm{H}$, Shiokawa $\mathrm{M}$, et al. Involvement of interleukin-17A-induced expression of heat shock protein 47 in intestinal fibrosis in Crohn's disease. Gut 2014; 63: 1902-1912.

13 Sato Y, Murase K, Kato J, et al. Resolution of liver cirrhosis using vitamin A-coupled liposomes to deliver siRNA against a collagen-specific chaperone. Nat Biotechnol 2008; 26: 431-442.

14 Otsuka M, Shiratori M, Chiba H, et al. Treatment of pulmonary fibrosis with siRNA against a collagen-specific chaperone HSP47 in vitamin A-coupled liposomes. Exp Lung Res 2017; 43: 271-282.

15 Yamakawa T, Ohigashi H, Hashimoto D, et al. Vitamin A-coupled liposomes containing siRNA against HSP47 ameliorate skin fibrosis in chronic graft-versus-host disease. Blood 2018; 131: 1476-1485.

16 Ohigashi $\mathrm{H}$, Hashimoto D, Hayase E, et al. Ocular instillation of vitamin A-coupled liposomes containing HSP47 siRNA ameliorates dry eye syndrome in chronic GVHD. Blood Adv 2019; 3: 1003-1010.

17 Kakugawa T, Mukae H, Hayashi T, et al. Expression of HSP47 in usual interstitial pneumonia and nonspecific interstitial pneumonia. Respir Res 2005; 6: 57-66.

18 Kakugawa T, Yokota S, Ishimatsu Y, et al. Serum heat shock protein 47 levels in patients with drug-induced lung disease. Respir Res 2013; 14: 133-143.

19 Iwashita T, Kadota J, Naito S, et al. "Involvement of collagen-binding heat shock protein 47 and procollagen type I synthesis in idiopathic pulmonary fibrosis: contribution of type II pneumocytes to fibrosis. Hum Pathol 2000; 31: 1498-1505.

20 A phase 1, randomized, double-blind, placebo-controlled escalating single dose study to evaluate the safety, tolerability and pharmacokinetics of ND-L02-s0201 injection in normal healthy subjects. https://www.clinicaltrials. gov/ct2/show/NCT01858935 Date last updated: May 11, 2017.

21 A study of safety, tolerability, and the effects two ND-L02-s0201 have on the body. www.clinicaltrials.gov/ct2/ show/NCT03241264 Date last updated: August 7, 2017.

22 Phase $1 \mathrm{~b} / 2$, open label, repeat dose, dose escalation study of ND L02-s0201 injection in subjects with moderate to extensive fibrosis (METAVIR F3-4). www.clinicaltrials.gov/ct2/show/NCT02227459 Date last updated: May 11, 2017.

23 JUNIPER: a phase 2 study to evaluate the safety, biological activity, and PK of ND-L02-s0201 in subjects with IPF www.clinicaltrials.gov/ct2/show/NCT03538301 Date last updated: June 18, 2020.

24 Degryse $\mathrm{A}$, Tanjore $\mathrm{H}, \mathrm{Xu} \mathrm{X}$, et al. Repetitive intratracheal bleomycin models several features of idiopathic pulmonary fibrosis. Am J Physiol Lung Cell Mol Physiol 2010; 299: 442-452.

25 Barbarin V, Nihoul A, Misson P, et al. The role of pro- and anti-inflammatory responses in silica-induced lung fibrosis. Respir Res 2005; 6: 112-125.

26 Hewitson T, Becker G. Kidney Research-experimental protocols. Springer, 2009; pp. 223-235, Vol. 466.

27 Bantikassegn A, Song X, Politi K. Isolation of epithelial, endothelial, and immune cells from lungs of transgenic mice with oncogene-induced lung adenocarcinomas. Am J Respir Cell Mol Biol 2015; 52: 409-417.

28 Gonzalez R, Dobbs L. Isolation and culture of alveolar epithelial Type I and Type II cells from rat lungs. Methods Mol Biol 2013; 945: 145-159.

29 Egger C, Cannet C, Gérard C, et al. Administration of bleomycin via the oropharyngeal aspiration route leads to sustained lung fibrosis in mice and rats as quantified by UTE-MRI and histology. PLoS One 2013; 8: e63432.

30 King TJ, Pardo A, Selman M. Idiopathic pulmonary fibrosis. Lancet 2011; 378: 1949-1961.

31 Amenomori M, Mukae H, Sakamoto N, et al. HSP47 in lung fibroblasts is a predictor of survival in fibrotic nonspecific interstitial pneumonia. Respir Med 2010; 104: 895-901.

32 Taguchi T, Razzaque M. "The collagen-specific molecular chaperone HSP47: is there a role in fibrosis? Trends Mol Med 2007; 13: 45-53.

33 Hinz B, Phan S, Thannickal V, et al. The myofibroblast: one function, multiple origins. Am J Pathol 2007; 170: $1807-1816$.

34 Kis K, Liu X, Hagood J. Myofibroblast differentiation and survial in fibrotic disease. Expert Rev Mol Med 2011; 13 e27.

35 Lim J, Thiery J. Epithelial-mesenchymal transitions: insights from development. Development 2012; 139: 3471-3486.

36 Miyamura T, Sakamoto N, Kakugawa $\mathrm{T}$, et al. Small molecule inhibitor of HSP47 prevents pro-fibrotic mechanisms of fibroblasts in vitro. Biochem Biophys Res Commun 2020; 530: 561-565.

37 Kawasaki K, Ushioda R, Ito S, et al. Deletion of the collagen-specific molecular chaperone Hsp47 causes endoplasmic reticulum stress-mediated apoptosis of hepatic stellate cells. J Biol Chem 2015; 290: 3639-3646.

38 Moeller A, Ask K, Warburton D, et al. The bleomycin animal model: a useful tool to investigate treatment options for idiopathic pulmonary fibrosis? Int J Biochem Cell Biol 2008; 40: 362-282.

39 Mouratis M, Aidinis V. Modeling pulmonary fibrosis with bleomycin. Curr Opin in Pulm Med 2011; 17: 355-361.

40 Ojo AS, Balogun SA, Williams OT, et al. Pulmonary fibrosis in COVID-19 survivors: predictive factors and risk reduction strategies. Pulmonary Medicine 2020; 2020: 6175964. 